Review

\title{
A Literature Review of Dietary Habits, Defecation Habits and Skill Care to Prevent Fecal Incontinence in Elderly Individuals with Irritable Bowel Syndrome
}

Yohei Okawa*, Ph.D., R.N.,

\author{
Department of Disability Science, Tohoku University Graduate School of Medicine. 2-1 Seiryo-machi, Aoba-ku, \\ Sendai, Miyagi, 980-8575, Japan. \\ * Correspondence: yohei-tky@umin.ac.jp; Tel.: +81-80-5483-3933 (Y.O.)
}

\begin{abstract}
The irritable bowel syndrome (IBS) is functional gastrointestinal tract disease, include abnormal defecation and abdominal pain. The Rome IV criteria define fecal incontinence as "recurrent and uncontrolled stool leakage that lasts more than 3 months." Fecal incontinence is common in patients with IBS and can have a significant negative impact on daily life and reduce the patient's quality of life. Diet and lifestyle guidance are needed to prevent fecal incontinence. Fecal incontinence can be reduced by ingesting dietary fiber, which can improve stool properties, and avoiding foods with stool-softening properties. Additionally, defecation habit guidance is important for preventing fecal incontinence. If rectal sensation is normal, it is recommended to go to the bathroom as soon as there is a desire to defecate. In elderly people, if there is stool in the rectum due to decreased rectal sensation and it continues to accumulate in the rectum without triggering the urge to defecate, overflowing leaky fecal incontinence may occur. For such patients, defecation habit training teaching them to defecate even if they do not have the desire to defecate may be effective. Education and advice on defecation reduces fecal incontinence and is beneficial to caregivers.
\end{abstract}

Keywords: fecal incontinence; unconscious elderly; irritable bowel syndrome; gastrointestinal symptoms; constipation; diarrhea

\section{Introduction}

Functional gastrointestinal disorders (FGIDs) are diseases in which gastrointestinal symptoms persist chronically or recurrently, laboratory tests show no organic lesions, and symptoms occur due to dysfunction [1]. Types of FGIDs include irritable bowel syndrome (IBS), functional abdominal distension, functional constipation, functional diarrhea, and unspecified functional bowel disease [1-3]. Symptoms of IBS, a typical functional gastrointestinal disorder, include abdominal pain, abdominal discomfort, and associated bowel abnormalities [3]. The Rome IV diagnostic criteria are used to diagnose IBS. According to the Rome IV diagnostic criteria, IBS can be diagnosed if "abdominal pain occurs at least one day per week in the last three months, and the abdominal pain is associated with bowel movements and associated with changes in bowel movement frequency" [3]. IBS patients are defined as "those with two or more symptoms of three bowel abnormalities related to changes in stool shape (appearance)". The Rome IV diagnostic criteria define fecal incontinence as "recurrent and uncontrolled stool leakage that lasts more than 3 months." Fecal incontinence can often occur in patients with IBS, and fecal incontinence can have a significant negative impact on daily life and reduce the patient's quality of life. 


\section{Defecation Disorders Due to IBS}

The Rome IV diagnostic criteria, which assess subjective symptoms, are used to diagnose IBS [1-4]. The Rome IV diagnostic criteria can distinguish between functional bowel disease with chronic symptoms and transient gastrointestinal symptoms. The criteria are as follows: "abdominal pain occurs at least one day per week in the last three months, and the abdominal pain is related to (1)bowel movements, (2)changes in bowel movement frequency, and (3) stool shape (appearance)." Functional bowel disease is defined as "change related, with two or more symptoms of three bowel movement abnormalities" that lasted more than 6 months before diagnosis and occurred more than 3 days in the last 3 months [3,4]. IBS subtypes can be divided into IBS with diarrhea (IBS-D), IBS with constipation (IBS-C), mixed IBS (IBS-M), and unclassifiable IBS (IBS-U). These subtypes are considered useful in clinical practice and treatment. Keep in mind that the Rome IV criteria assess only subjective symptoms, making it difficult to diagnose unconscious or cognitively impaired patients. In addition, IBS stool shapes vary from watery stools to hard stools, suggesting that the time it takes to pass through the gastrointestinal tract is reflected by the stool shape [5,6]. On the other hand, it has also been reported that defecation frequency and transit time in IBS patients are often the same as those in healthy subjects $[7,8]$. IBS causes intestinal motility disorders such as constipation and diarrhea and abnormal bowel movements, although defecation symptoms differ depending on each subtype. Therefore, IBS is a clear high-risk factor for fecal incontinence [9-11].

\section{Definition of Fecal Incontinence}

IBS is often a high-risk factor for fecal incontinence [9-11]. The definition of stool disease varies slightly from country to country. The International Consultation on Incontinence (ICI) defines anal incontinence and fecal incontinence separately. Anal incontinence is defined as "the involuntary loss of flatus, liquid or solid stool that is a social or hygienic problem." Fecal incontinence is defined as "the involuntary loss of liquid or solid stool that is a social or hygienic problem"; therefore, fecal incontinence is defined as anal incontinence minus flatus incontinence $[12,13]$.

On the other hand, the American Society of Colon and Rectal Surgeons (ASCRS) defines anal incontinence as "the uncontrolled passage of feces or gas over at least 1 month's duration, in an individual of at least 4 years of age, who had previously achieved control". Age and duration of illness are included in the definition without distinguishing between fecal incontinence and flatus incontinence [14-16]. In addition, the American College of Gastroenterology (ACG) defines anal incontinence as "either the involuntary passage or the inability to control the discharge of fecal matter through the anus" [17], and the assessment includes variables such as social background, age, and duration of illness. Only flatus incontinence is excluded.

In this review, fecal incontinence is defined as "a symptom of stool leaking from the anus unconsciously or against one's will". Flatus incontinence is defined as "a symptom of gas leaking from the anus unconsciously or against one's will." Fecal incontinence and flatus incontinence are collectively defined as anal incontinence.

\section{Classification and Assessment of Fecal Incontinence}

Fecal incontinence can be divided into urgent fecal incontinence and leaky fecal incontinence or mixed fecal incontinence, which is a mixture of both [17]. Imminent fecal incontinence is a "symptom of feeling stool but not being able to reach a restroom in time." Leaky fecal incontinence is a "symptom of fecal incontinence that occurs without being noticed." Fecal incontinence is rarely caused by a single factor, and multiple factors are often interrelated [18]. Therefore, fecal incontinence does not necessarily mean that the anal sphincter is impaired. If fecal incontinence is caused by a disorder of the anal sphincter, a decrease in internal anal sphincter function reduces the anal canal resting pressure, resulting in leaky fecal incontinence. When the external anal sphincter is weakened, the 
voluntary contraction pressure of the anal canal is reduced, and urge incontinence is likely to occur [19]. On the other hand, even if the anal sphincter is normal, the rectal sensation may be reduced, and even if there is feces in the rectum, the sensation of stool may not be felt. Then, fecal embolism may occur, resulting in overflowing fecal incontinence.

Irritable bowel incontinence may occur in IBS patients with increased rectal sensation and contractility, even if the anal sphincter is normal [20]. Diarrhea causes irritable bowel incontinence, depending on the stool properties. In addition, decreased rectal sensation due to IBS-C with hard stools and functional constipation may cause leaky fecal incontinence. Since the status of defecation varies greatly from individual to individual, it is important to understand individual daily defecation habits and their changes, and the Bristol stool property scale is used to evaluate stool properties [21]. As factors in daily life related to the onset of fecal incontinence in IBS patients, it is necessary to assess not only the general condition, including body movement restriction, individual cognitive ability, and basic activity, but also the living environment, including the toilet environment [18,22]. In addition, coexisting diseases other than IBS may be involved in the development of fecal incontinence. Taking therapeutics and laxatives for comorbidities often also causes fecal incontinence. Psychotic drugs may act on intestinal motility and peripheral nerves to cause fecal incontinence [23]. In addition, coffee and alcohol affect intestinal motility and stool properties, and smoking history plays a role in the atrophy of the external anal sphincter muscle and causes urgent fecal incontinence [22]. In summary, fecal incontinence includes urgent fecal incontinence, leaky fecal incontinence, and mixed fecal incontinence, and it is important to understand the factors related to each. In particular, IBS is associated with different fecal properties depending on the subtype and may cause various types of fecal incontinence. Therefore, it is necessary to fully assess the daily lifestyle, defecation habits, and eating habits of IBS patients.

\section{Questionnaire for the Clinical Evaluation of IBS-related Fecal Incontinence}

The diagnosis of IBS is based on the Rome IV diagnostic criteria [4] and other questionnaires. However, because of the many factors that contribute to the development of IBS, several questionnaires are used to assess the relationship between daily living conditions, quality of life (QOL), psychological status, and abdominal symptoms. [23-26]. The evaluation of QOL using the SF-36 is clinically useful because it can be compared with QOL information from patients with other diseases. In addition, IBS-QOL, an IBS-specific QOL study, is effective in assessing the effectiveness of treatment [27]. The SCL-90-R, HDRS [28], EPQ, and DSSI [23] questionnaires are also available. Symptoms of IBS can be assessed by combining these questionnaires. In particular, questionnaires can reveal mental problems such as anxiety and depression. Several questionnaires are used for the clinical evaluation of fecal incontinence. The Rome IV diagnostic criteria define fecal incontinence as "recurrent and uncontrolled stool leakage that lasts more than 3 months." The use of questionnaires is useful because fecal incontinence can often occur in patients with IBS, and fecal incontinence can have a significant negative impact on daily life and reduce the patient's QOL [29]. The severity criteria for fecal incontinence include the Kirwan classification [30], Miller score [31], Pescatori score [32], Cleveland Clinic Florida Fecal Incontinence score (CCFIS) [33], St. Mark's score [34], the Fecal Incontinence Severity Index (FISI) [35], etc. In recent years, the CCFIS has often been used for fecal incontinence symptoms and QOL evaluation. In addition, the St. Mark's score is often used when the sense of urgency is important.

It is suggested that clinical evaluation using these questionnaires is useful for medical treatment of IBS with fecal incontinence.

\section{Dietary Habits, Defecation Habits and Skill Care to Prevent Fecal Incontinence}

As dietary and lifestyle guidance for patients with fecal incontinence, patients should be instructed to refrain from ingesting caffeine, citrus fruits, spicy foods, and alcohol, 
which have the effect of softening the stool [36]. Dietary fiber, such as psyllium, has been reported to reduce fecal incontinence by improving stool properties [37]. It has also been reported that ingestion of dietary fiber in addition to taking antidiarrheal agents such as loperamide hydrochloride improved fecal incontinence [38]. On the other hand, a study that guided changes in diet and fluid intake in elderly stroke patients with reduced physical fitness to regulate bowel movements showed that normal bowel movements increased, but fecal incontinence remained significant and did not improve [39].

Defecation habit guidance is an important factor in preventing fecal incontinence. If the rectal sensation is normal, it is recommended to go to the bathroom as soon as possible once there is a desire to defecate. On the other hand, if the rectal sensation is reduced, fecal incontinence can be significantly improved by systematically trying to defecate even if there is no desire to defecate $[40,41]$. In elderly people, if there is stool in the rectum due to decreased rectal sensation and stool continues to accumulate in the rectum without triggering the urge to defecate, overflowing leaky fecal incontinence may occur. For such patients, defecation habit training may be effective in which twice a day (approximately 30 minutes after breakfast or dinner), the individual goes to the toilet to defecate even if there is no desire to defecate (stress defecation) $[40,41]$.

Educational guidance and advice on defecation from nurses reduces fecal incontinence and is also beneficial to caregivers [41]. In the context of poorly managed fecal incontinence, dermatitis such as erythema, erosion, and ulcers on the skin of the buttocks occurs. Moisturizing and protective skin care methods with mildly acidic cleansers and skin dressings reduce the incidence of fecal incontinence-related dermatitis [41].

In summary, dietary habits, defecation habits and skill care to prevent fecal incontinence are important and should be actively implemented.

\section{Can Fecal Incontinence in Unconscious Patients be Predicted and Prevented in Ad- vance?}

The Rome IV diagnostic criteria and other questionnaires are used to assess the patient's subjective symptoms, so they are not able to diagnose unconscious or cognitively impaired patients. Therefore, we will consider whether objective indicators can be used to predict and prevent fecal incontinence in unconscious patients in advance. In recent years, it has been reported that fecal retention can be objectively and noninvasively evaluated using ultrasound imaging equipment [42-45]. In addition, another previous study using ultrasound reported that gallbladder contraction was higher in the IBS group than in the control group, both in the fasted state and after dietary stress $[46,47]$. Although IBS has been suggested to be associated with cholecystectomy, the number of cases is small, and its direct association with IBS symptoms is unknown. In addition, a previous study in Japan reported that abdominal ultrasonography assessed colon motility, and observation of the sigmoid colon on an empty stomach revealed enhanced colonic contraction in the IBS group. Postprandial observations of the sigmoid colon compared nine IBS cases diagnosed according to the Rome II criteria with four controls. Segmentation was enhanced in IBS-C patients. On the other hand, IBS-D was observed to enhance the transport of intestinal contents to the anus [48]. Based on these reports, ultrasonography can assess defecation disorders as a noninvasive examination and may help assess bowel motility abnormalities that cause IBS-related fecal incontinence. However, since there are few research reports, future verification is required.

Other studies have performed transvaginal ultrasonography and objective evaluation of IBS using transrectal ultrasound [49-52]. However, transvaginal ultrasonography is a difficult method for people who lead a general healthy social life $[49,50]$. Since it is a painful examination method, it is difficult to use it in home medical care or medical facilities because it requires medical equipment.

Therefore, it is currently difficult to objectively evaluate IBS, and it is considered most important to fully evaluate the patient's chief complaint to prevent fecal incontinence 
related to IBS. Given this, predicting IBS-related fecal incontinence in advance using objective indicators should be considered in the future.

\section{Limitations}

The Rome IV diagnostic criteria based on subjective symptoms are used to diagnose IBS. This approach is limited to those who can communicate and describe their symptoms. Ultrasound, on the other hand, can be a tool for diagnosing IBS with objective indicators. To date, several previous studies have reported that constipation and normal stools can be easily and noninvasively identified percutaneously. However, using ultrasound, it is difficult to evaluate abdominal pain, which is indispensable for diagnosing IBS. In the future, it is necessary to consider methods for further visualization of defecation disorders and indicators that can objectively evaluate abdominal symptoms such as abdominal pain.

\section{Conclusion}

FGIDs are disorders in which gastrointestinal symptoms persist chronically or recurrently. In individuals with FGIDs, laboratory tests show no organic lesions, and symptoms are due to dysfunction. IBS is a typical FGID. Fecal incontinence can often occur in patients with IBS, and fecal incontinence can have a significant negative impact on daily life and reduce the patient's QOL. To prevent fecal incontinence, it is necessary to provide diet and lifestyle guidance. It has been reported that fecal incontinence can be reduced by ingesting dietary fiber, which can improve stool properties, and limit the ingestion of foods that have the effect of softening stool. There are also reports that fecal incontinence was improved by taking antidiarrheal agents. In addition, defecation habit guidance is an important factor in preventing fecal incontinence. If the rectal sensation is normal, it is recommended to go to the bathroom as soon as possible once there is a desire to defecate. On the other hand, if the rectal sensation is reduced, fecal incontinence can be significantly improved by systematically trying to defecate even if there is no desire to defecate. In particular, in elderly people, if there is stool in the rectum due to decreased rectal sensation and stool continues to accumulate in the rectum without triggering the urge to defecate, overflowing leaky fecal incontinence may occur. For such patients, defecation habit training that teaches them to go to the toilet and defecate even if they do not have the desire to defecate may be effective. Educational guidance and advice on defecation from nurses reduces fecal incontinence and is also beneficial to caregivers. In the context of poorly managed fecal incontinence, dermatitis such as erythema, erosion, and ulcers on the skin of the buttocks occurs. Moisturizing and protective skin care methods with mildly acidic cleansers and skin dressings reduce the incidence of dermatitis associated with fecal incontinence. In conclusion, it is considered that dietary habits, defecation habits and skill care to prevent fecal incontinence are important and should be actively implemented.

\section{Acknowledgments}

We would like to express our sincere gratitude to the reviewers for providing valuable information in the process of writing this article.

\section{Author Contributions}

The author contributed to the data collection, manuscript preparation and completed the final manuscript.

\section{Funding}

The study did not receive funding. 


\title{
Institutional Review Board Statement
}

This study was conducted as part of a pilot study.

\author{
Informed Consent Statement \\ Not applicable.
}

\section{Data Availability Statement}

The data presented in this study are available on request from the corresponding author.

\section{Conflicts of Interest}

The author declare that we have no competing interests.

\section{References}

1. Longstreth, G.F.; Thompson, W.G.; Chey, W.D.; Houghton, L.A.; Mearin, F.; Spiller, R.C. Functional bowel disorders. Gastroenterology 2006, 130, 1480-1491; DOI:10.1053/j.gastro.2005.11.061.

2. Drossman, D.A. Functional gastrointestinal disorders: History, pathophysiology, clinical features, and Rome IV. Gastroenterology 2016, 150, 1262-1279.e1262; DOI:10.1053/j.gastro.2016.02.032.

3. Drossman, D.A. The functional gastrointestinal disorders and the Rome III process. Gastroenterology 2006, 130, 1377-1390; DOI:10.1053/j.gastro.2006.03.008.

4. Drossman, D.A.; Hasler, W.L. Rome IV-Functional GI disorders: Disorders of gut-brain interaction. Gastroenterology 2016, 150, 1257-1261; DOI:10.1053/j.gastro.2016.03.035.

5. Degen, L.P.; Phillips, S.F. How well does stool form reflect colonic transit? Gut 1996, 39, 109-113; DOI:10.1136/gut.39.1.109.

6. Törnblom, H.; Van Oudenhove, L.; Sadik, R.; Abrahamsson, H.; Tack, J.; Simrén, M. Colonic transit time and IBS symptoms: What's the link? Am. J. Gastroenterol. 2012, 107, 754-760; DOI:10.1038/ajg.2012.5.

7. Ragnarsson, G.; Bodemar, G. Division of the irritable bowel syndrome into subgroups on the basis of daily recorded symptoms in two outpatient samples. Scand. J. Gastroenterol. 1999, 34, 993-1000; DOI:10.1080/003655299750025093.

8. Saad, R.J.; Rao, S.S.C.; Koch, K.L.; Kuo, B.; Parkman, H.P.; McCallum, R.W.; Sitrin, M.D.; Wilding, G.E.; Semler, J.R.; Chey, W.D. Do stool form and frequency correlate with whole-gut and colonic transit? Results from a multicenter study in constipated individuals and healthy controls. Am. J. Gastroenterol. 2010, 105, 403-411; DOI:10.1038/ajg.2009.612.

9. Burgell, R.E.; Bhan, C.; Lunniss, P.J.; Scott, S.M. Fecal incontinence in men. Dis. Colon Rectum 2012, 55, 18-25; DOI:10.1097/dcr.0b013e318237f37d.

10. Scarlett, Y. Medical management of fecal incontinence. Gastroenterology 2004, 126, S55-S63; DOI:10.1053/j.gastro.2003.10.007.

11. Varma, M.G.; Brown, J.S.; Creasman, J.M.; Thom, D.H.; Van Den Eeden, S.K.; Beattie, M.S.; Subak, L.L.; Reproductive Risks for Incontinence Study at Kaiser Research Group. Fecal incontinence in females older than aged 40 years: Who is at risk? Dis. Colon Rectum 2006, 49, 841-851; DOI:10.1007/s10350-006-0535-0.

12. Norton, C.; Whitehead, W.E.; Bliss, D.Z.; Harari, D.; Lang, J. Conservative and pharmacological management of fecal incontinence in adults. In Incontinence; Abrams, P., Cardoza, L., Robinson, D., Miles, A., Eds.; Plybridge: London, UK, 2005; pp. 15211563.

13. Whitehead, W.E.; Wald, A.; Norton, N.J. Treatment options for fecal incontinence. Dis. Colon Rectum 2001, 44, 131-142; DOI:10.1007/bf02234835.

14. Tjandra, J.J.; Dykes, S.L.; Kumar, R.R.; Ellis, C.N.; Gregorcyk, S.G.; Hyman, N.H.; Buie, W.D. Practice parameters for the treatment of fecal incontinence. Dis. Colon Rectum 2007, 50, 1497-1507; DOI:10.1007/s10350-007-9001-x.

15. Wald, A. Fecal incontinence in adults. N. Engl. J. Med. 2007, 356, 1648-1655; DOI:10.1056/nejmcp067041.

16. Madoff, R.D.; Parker, S.C.; Varma, M.G.; Lowry, A.C. Faecal incontinence in adults. Lancet 2004, 364, 621-632; DOI:10.1016/s0140-6736(04)16856-6.

17. Rao, S.S.C. Diagnosis and management of fecal incontinence. Am. J. Gastroenterol. 2004, 99, 1585-1604; DOI:10.1111/j.15720241.2004.40105.x.

18. Wu, J.M.; Matthews, C.A.; Vaughan, C.P.; Markland, A.D. Urinary, fecal, and dual incontinence in older U.S. adults. J. Am. Geriatr. Soc. 2015, 63, 947-953; DOI:10.1111/jgs.13385.

19. Engel, A.F.; Kamm, M.A.; Bartram, C.I.; Nicholls, R.J. Relationship of symptoms in faecal incontinence to specific sphincter abnormalities. Int. J. Colorectal Dis. 1995, 10, 152-155; DOI:10.1007/bf00298538.

20. Hawkins, A.T.; Olariu, A.G.; Savitt, L.R.; Gingipally, S.; Wakamatsu, M.M.; Pulliam, S.; Weinstein, M.M.; Bordeianou, L. Impact of rising grades of internal rectal intussusception on fecal continence and symptoms of constipation. Dis. Colon Rectum 2016, 59, 54-61; DOI:10.1097/dcr.0000000000000510. 
21. Wald, A.; Bharucha, A.E.; Cosman, B.C.; Whitehead, W.E. ACG clinical guideline: Management of benign anorectal disorders. Am. J. Gastroenterol. 2014, 109, 1141-1157; DOI:10.1038/ajg.2014.190.

22. Townsend, M.K.; Matthews, C.A.; Whitehead, W.E.; Grodstein, F. Risk factors for fecal incontinence in older women. Am. J. Gastroenterol. 2013, 108, 113-119; DOI:10.1038/ajg.2012.364.

23. Boyce, P.M.; Koloski, N.A.; Talley, N.J. Irritable bowel syndrome according to varying diagnostic criteria: Are the new Rome II criteria unnecessarily restrictive for research and practice? Am. J. Gastroenterol. 2000, 95, 3176-3183; DOI:10.1111/j.15720241.2000.03197.x.

24. Walter, S.A.; Kjellström, L.; Talley, N.J.; Andreasson, A.N.; Nyhlin, H.; Agréus, L. Prospective diary evaluation of unexplained abdominal pain and bowel dysfunction: A population-based colonoscopy study. Dig. Dis. Sci. 2011, 56, 1444-1451; DOI:10.1007/s10620-010-1468-y.

25. Robinson, A.; Lee, V.; Kennedy, A.; Middleton, L.; Rogers, A.; Thompson, D.G.; Reeves, D. A randomised controlled trial of self-help interventions in patients with a primary care diagnosis of irritable bowel syndrome. Gut 2006, 55, 643-648; DOI:10.1136/gut.2004.062901.

26. Andrae, D.A.; Patrick, D.L.; Drossman, D.A.; Covington, P.S. Evaluation of the Irritable Bowel Syndrome Quality of Life (IBSQOL) questionnaire in diarrheal-predominant irritable bowel syndrome patients. Health Qual. Life Outcomes 2013, 11, 208; DOI:10.1186/1477-7525-11-208.

27. Heitkemper, M.M.; Jarrett, M.E.; Levy, R.L.; Cain, K.C.; Burr, R.L.; Feld, A.; Barney, P.; Weisman, P. Self-management for women with irritable bowel syndrome. Clin. Gastroenterol. Hepatol. 2004, 2, 585-596; DOI:10.1016/s1542-3565(04)00242-3.

28. Guthrie, E.; Creed, F.; Fernandes, L.; Ratcliffe, J.; Van Der Jagt, J.; Martin, J.; Howlett, S.; Read, N.; Barlow, J.; Thompson, D.; et al. Cluster analysis of symptoms and health seeking behaviour differentiates subgroups of patients with severe irritable bowel syndrome. Gut 2003, 52, 1616-1622; DOI:10.1136/gut.52.11.1616.

29. Paquette, I.M.; Varma, M.G.; Kaiser, A.M.; Steele, S.R.; Rafferty, J.F. The American Society of colon and rectal surgeons' clinical practice guideline for the treatment of fecal incontinence. Dis. Colon Rectum 2015, 58, 623-636; DOI:10.1097/dcr.0000000000000397.

30. Kirwan, W.O.; Turnbull, R.B.; Fazio, V.W.; Weakley, F.L. Pullthrough operation with delayed anastomosis for rectal cancer. Br. J. Surg. 1978, 65, 695-698; DOI:10.1002/bjs.1800651008.

31. Miller, R.; Bartolo, D.C.C.; Locke-Edmunds, J.C.; McC. Mortensen, N.J. Prospective study of conservative and operative treatment for faecal incontinence. Br. J. Surg. 1988, 75, 101-105; DOI:10.1002/bjs.1800750204.

32. Pescatori, M.; Anastasio, G.; Bottini, C.; Mentasti, A. New grading and scoring for anal incontinence. Dis. Colon Rectum 1992, 35, 482-487; DOI:10.1007/bf02049407.

33. Jorge, M.J.N.; Wexner, S.D. Etiology and management of fecal incontinence. Dis. Colon Rectum 1993, 36, 77-97; DOI:10.1007/bf02050307.

34. Vaizey, C.J.; Carapeti, E.; Cahill, J.A.; Kamm, M.A. Prospective comparison of faecal incontinence grading systems. Gut 1999, 44, 77-80; DOI:10.1136/gut.44.1.77.

35. Rockwood, T.H.; Church, J.M.; Fleshman, J.W.; Kane, R.L.; Mavrantonis, C.; Thorson, A.G.; Wexner, S.D.; Bliss, D.; Lowry, A.C. Patient and surgeon ranking of the severity of symptoms associated with fecal incontinence. Dis. Colon Rectum 1999, 42, 15251531; DOI:10.1007/bf02236199.

36. Rao, S.S.C. Current and emerging treatment options for fecal incontinence. J. Clin. Gastroenterol. 2014, 48, 752-764; DOI:10.1097/MCG.0000000000000180.

37. Bliss, D.Z.; Savik, K.; Jung, H.-J.G.; Whitebird, R.; Lowry, A.; Sheng, X. Dietary fiber supplementation for fecal incontinence: A randomized clinical trial. Res. Nurs. Health 2014, 37, 367-378; DOI:10.1002/nur.21616.

38. Lauti, M.; Scott, D.; Thompson-Fawcett, M.W. Fibre supplementation in addition to loperamide for faecal incontinence in adults: A randomized trial. Colorectal Dis. 2008, 10, 553-562; DOI:10.1111/j.1463-1318.2007.01439.x.

39. Harari, D.; Norton, C.; Lockwood, L.; Swift, C. Treatment of constipation and fecal incontinence in stroke patients. Stroke 2004, 35, 2549-2555; DOI:10.1161/01.str.0000144684.46826.62.

40. Norton, C.; Whitehead, W.E.; Bliss, D.Z.; Harari, D.; Lang, J. Management of fecal incontinence in adults. Neurourol. Urodyn. 2010, 29, 199-206; DOI:10.1002/nau.20803.

41. Bliss, D.Z.; Norton, C. Conservative management of fecal incontinence. Am. J. Nurs. 2010, 110, 30-38; DOI:10.1097/01.naj.0000388262.72298.f5.

42. Yabunaka, K.; Matsumoto, M.; Yoshida, M.; Tanaka, S.; Miura, Y.; Tsutaoka, T.; Handa, M.; Nakagami, G.; Sugama, J.; Okada, S.; et al. Assessment of rectal feces storage condition by a point-of-care pocket-size ultrasound device for healthy adult subjects: A preliminary study. Drug Discov. Ther. 2018, 12, 42-46; DOI:10.5582/ddt.2018.01001.

43. Tanaka, S.; Yabunaka, K.; Matsumoto, M.; Tamai, N.; Noguchi, H.; Yoshida, M.; Nakagami, G.; Sugama, J.; Sanada, H. Fecal distribution changes using colorectal ultrasonography in older people with physical and cognitive impairment living in longterm care facilities: A longitudinal observational study. Healthcare (Basel, Switzerland) 2018, 6, 55; DOI:10.3390/healthcare6020055.

44. Matsumoto, M.; Tanaka, S.; Yabunaka, K.; Yoshida, M.; Miura, Y.; Tsutaoka, T.; Handa, M.; Nakagami, G.; Sugama, J.; Okada, S.; et al. Ultrasonographic evaluation of changes over time in one defecation cycle in adults with functional constipation: A report of two cases. Drug Discov. Ther. 2018, 12, 304-308; DOI:10.5582/ddt.2018.01054. 
45. Yabunaka, K.; Nakagami, G.; Tabata, K.; Sugama, J.; Matsumoto, M.; Kido, Y.; Iuchi, T.; Sanada, H. Constipation in the elderly in a Japanese long-term medical facility: An ultrasonographic investigation. Drug Discov. Ther. 2018, 12, 233-238; DOI:10.5582/ddt.2018.01033.

46. Guliter, S.; Yilmaz, S.; Yakaryilmaz, F.; Keles, H. Evaluation of gallbladder motility in patients with irritable bowel syndrome. Swiss. Med. Wkly. 2005, 135, 407-411.

47. Guclu, M.; Pourbagher, A.; Serin, E.; Kul, K.; Ozer, B.; Cosar, A.; Icer, M.O.; Gur, G.; Boyacioglu, S. Ultrasonographic evaluation of gallbladder functions in patients with irritable bowel syndrome. J. Gastroenterol. Hepatol. 2006, 21, 1309-1312; DOI:10.1111/j.1440-1746.2006.04136.x.

48. Kusunoki, H.; Kamada, T.; Sato, M.; Haruma, K.; Hata, J. Ultrasonographic assessment of sigmoid colon in patients with irritable bowel syndrome. Nihon Rinsho 2006, 64, 1461-1466.

49. Devanarayana, N.M.; Rajindrajith, S.; Bandara, C.; Shashiprabha, G.; Benninga, M.A. Ultrasonographic assessment of liquid gastric emptying and antral motility according to the subtypes of irritable bowel syndrome in children. J. Pediatr. Gastroenterol. Nutr. 2013, 56, 443-448; DOI:10.1097/mpg.0b013e31827f7a3d.

50. Crade, M.; Pham, V. Ultrasound examination of the sigmoid colon: Possible new diagnostic tool for irritable bowel syndrome. Ultrasound Obstet. Gynecol. 2006, 27, 206-209; DOI:10.1002/uog.2620.

51. Awad, R.A.; Martin, J.; Cal y Major, M.; Noguera, J.L.; Ramos, R.; Amezcua, C.; Camacho, S.; Santiago, R.; Ramirez, J.L.; Castro, J. Transrectal ultrasonography: Relationship with anorectal manometry, electromyography and sensitivity tests in irritable bowel syndrome. Int. J. Colorectal Dis. 1998, 13, 82-87; DOI:10.1007/s003840050140.

52. O'Connor, O.J.; McSweeney, S.E.; McWilliams, S.; O’Neill, S.; Shanahan, F.; Quigley, E.M.M.; Maher, M.M. Role of radiologic imaging in irritable bowel syndrome: Evidence-based review. Radiology 2012, 262, 485-494; DOI:10.1148/radiol.11110423. 